\title{
Innovation von datengetriebenen Geschäftsmodellen
}

\section{Oft probiert, häufig gescheitert? Ein Vorgehensmodell zur Wertrealisierung von Daten}

\author{
Matthias Förster · Bastian Bansemir • Angela Roth (iD
}

Eingegangen: 30. Dezember 2020 / Angenommen: 29. März 2021 / Online publiziert: 21. April 2021

(C) Der/die Autor(en) 2021

Zusammenfassung Im Rahmen der fortschreitenden Digitalisierung ist die Wettbewerbsfähigkeit von Unternehmen zunehmend von der Fähigkeit abhängig, datengetriebene Geschäftsmodelle zu innovieren. Jedoch stellt dies Unternehmen vor große Herausforderungen, und so bleiben datengetriebene Geschäftsmodelle nach der Implementierung am Markt nicht selten hinter den Erwartungen an die Wertrealisierung, z. B. in Form von Umsatz oder Kosteneinsparung zurück. Dies liegt insbesondere daran, dass sich wertrealisierende oder wertmindernde Einflussfaktoren häufig erst nach der Implementierung am Markt zeigen. Unternehmen fehlen hier konkrete Vorgehensmodelle, um gezielt diesen Einflussfaktoren zu begegnen und datengetriebene Geschäftsmodellinnovationen erfolgreich umzusetzen. Die vorliegende Studie greift diese Thematik auf und entwickelt im Rahmen eines Design Science Forschungsansatzes ein interview- und workshopbasiertes Vorgehensmodell, welches Praktiker bei der Realisierung von Werten bei der Implementierung von datengetriebenen Geschäftsmodellen unterstützt. Die beispielhafte Anwendung des Vorgehensmodells im Rahmen von fünf datengetriebenen Geschäftsmodellen eines deutschen Premiumautomobilherstellers zeigt, dass dadurch wertmindernde und wertrealisierende Einflussfaktoren identifiziert und bewertet werden können. Zudem können eine fachbereichsübergreifende Definition von Wertrealisierung, ein holisti-

\footnotetext{
M. Förster · A. Roth $(\bowtie)$

Wirtschaftsinformatik, insb. Innovation \& Wertschöpfung, Friedrich-Alexander Universität Erlangen-Nürnberg, Lange Gasse 20, 90403 Nürnberg, Deutschland

E-Mail: angela.roth@fau.de

M. Förster

E-Mail: matthias.f.foerster@fau.de

B. Bansemir

Business Model Development, BMW Group, München, Deutschland

E-Mail: bastian.bansemir@bmw.de
} 
sches Verständnis über die entscheidenden Einflussfaktoren und ihre Hebelwirkung sowie Kenntnisse über kausale Zusammenhänge zwischen zu ergreifenden Maßnahmen und der Wertrealisierung entwickelt werden. Auch die Verteilung begrenzter Unternehmensressourcen für die Umsetzung von Maßnahmen in Relation zu deren Effekten wird explizit berücksichtigt.

Schlüsselwörter Datengetriebene Geschäftsmodelle · Vorgehensmodell zur Wertrealisierung · Innovation · Design Science

\title{
Innovation of Data-Driven Business Models
}

A Procedure Model for Value Realization

\begin{abstract}
In the context of the ongoing digitalization, the competitiveness of companies is increasingly dependent on their ability to innovate data-driven business models. However, this poses major challenges for companies, and so, data-driven business models, once they have been implemented on the market, often fall short of expectations in terms of value realization, e.g. in the form of revenues or cost savings. In particular, this is due to the fact that value-realizing or value-reducing influencing factors often only become apparent after the implementation in the market. At this point, companies lack concrete procedure models to counter these influencing factors and to successfully implement data-driven business models and realize value. By means of a design science research approach, this study develops an interview- and workshop-based procedure model that enables practitioners to implement and realize value from data-driven business models. The application of the procedure model in five data-driven business models of a German premium car manufacturer has shown that it offers practitioners useful support. For example, it provides an easy-to-understand methodological approach that helps to identify and evaluate value-realizing and value-reducing influencing factors, promote crossfunctional, shared knowledge on value realization, decision factors and the leveraging effects of different measures. Additionally, the assignment of limited company resources to the implementation of different measures in relation to their effects is considered.
\end{abstract}

Keywords Data-driven business models - Procedure model for value realization Innovation · Design science research

\section{Einleitung}

Innovationen von datengetriebenen Geschäftsmodellen spielen eine entscheidende Rolle für die Wettbewerbsfähigkeit von Unternehmen. Inzwischen werden datengetriebene Geschäftsmodelle in einer Vielzahl von Branchen erfolgreich eingesetzt, z.B. in der Automobilbranche (Bosler et al. 2018) oder in der Logistik (Möller et al. 2020) und in weiteren industriellen und informationstechnischen Bereichen (Oberländer et al. 2019). Wie sich jedoch aus Daten im Kontext datengetriebener Geschäftsmodelle langfristig Wert für ein Unternehmen bzw. dessen Kunden rea- 
lisieren lässt, ist in vielerlei Hinsicht noch nicht erforscht. Weder der Besitz oder Zugang noch die Nutzung von Daten sind per se ein Garant für die Realisierung von Werten in Unternehmen und so schaffen es viele Unternehmen nicht, trotz aktiver Datennutzung im Rahmen datengetriebener Geschäftsmodelle, Werte zu generieren (Günther et al. 2017). Die Wertrealisierung, z. B. in Form von Umsatzerhöhung oder Kosteneinsparung, bleibt nach der Implementierung der jeweiligen datengetriebenen Geschäftsmodelle häufig hinter den Erwartungen zurück. Wertrealisierende oder wertmindernde Einflussfaktoren zeigen sich dabei erst nach der Implementierung datengetriebener Geschäftsmodelle und müssen entsprechend kontinuierlich gemanagt werden, um nachhaltigen Erfolg zu ermöglichen. Trotz erster vielversprechender Ansätze (siehe z. B. Kühne und Böhmann (2019) oder Kühne et al. (2019)) ist die Wertrealisierung datengetriebener Geschäftsmodelle insbesondere hinsichtlich der praktischen Umsetzung noch nicht umfassend verstanden. Insbesondere fehlen methodische Ansätze, wie z. B. Vorgehensmodelle, die Unternehmen gezielt in die Lage versetzen, aus datengetriebenen Geschäftsmodellen Werte zu generieren.

Der vorliegende Beitrag präsentiert ein mittels Design Science Research (Hevner et al. 2004) entwickeltes interview- und workshopbasiertes Vorgehensmodell, um praktischen Herausforderungen bei der Realisierung von Werten im Kontext der Innovation datengetriebener Geschäftsmodelle begegnen und letztere erfolgreich implementieren zu können. In Abschn. 2 wird zunächst auf die theoretischen Grundlagen zu datengetriebenen Geschäftsmodellen und ihrer Wertrealisierung eingegangen. Anschließend wird in Abschn. 3 die Entwicklung des Vorgehensmodells erörtert. Die Anwendung dieses Modells wird in Abschn. 4 anhand von fünf datengetriebenen Geschäftsmodellen eines deutschen Premiumautomobilherstellers beispielhaft demonstriert und evaluiert. Nach der beispielhaften Anwendung und Evaluation des Vorgehensmodells werden in Abschn. 5 die Implikationen für Forschung und Praxis diskutiert.

\section{Wertrealisierung in datengetriebenen Geschäftsmodellen}

Exponentielles Datenwachstum (Chen et al. 2012) und der anhaltende technologische Fortschritt im Bereich Datenanalyse ermöglichen die Erhaltung und den weiteren Ausbau der Wettbewerbsfähigkeit von Unternehmen durch sogenannte datengetriebene Geschäftsmodelle (Wiener et al. 2020). Diese können dabei als ineinandergreifende Systeme aus Akteuren und Beziehungen, Kosten und Erlösen sowie Prozessen, Strukturen, Aktivitäten und Fähigkeiten, die zusammengenommen ökonomische Werte für das Unternehmen durch neue Wertversprechen realisieren, definiert werden (siehe Literaturstudie von Hilbig et al. (2020)). Entsprechend können in einem Unternehmen auch mehrere datengetriebene Geschäftsmodelle nebeneinanderstehen. Daten stellen in datengetriebenen Geschäftsmodellen die Schlüsselressourcen dar (Hartmann et al. 2016). Die Wertrealisierung von Daten bezieht sich dabei sowohl auf den Entstehungsprozess von Wert in datenbasierten Geschäftsmodellen als auch auf dessen Realisierung oder auch Monetarisierung. Sie erfolgt durch: (1) die Nutzung von Daten zur Produktivitätsoptimierung von Geschäftsprozessen oder der Erneuerung der Wertarchitektur, z.B. durch neue Kernaktivitäten 
wie Datenanalyse und -visualisierung zur Einsparung von Kosten (Hartmann et al. 2016), (2) das Anbieten neuer Wertversprechen, die dem Kunden einen zusätzlichen Mehrwert bieten und somit zu vergleichsweise mehr Umsätzen führen oder (3) durch zusätzliche Umsätze aus neuartigen Erlösmodellen wie Datennutzungsgebühren (Schüritz et al. 2017).

Um in datengetriebenen Geschäftsmodellen Wert zu realisieren, z. B. durch die Erhebung und Lizensierung von aggregierten und analysierten Datenprodukten (Wixom und Ross 2017), benötigen Unternehmen neue Fähigkeiten und Kenntnisse wie zum Beispiel über den wertrealisierenden Einsatz von Data Analytics (Akter und Wamba 2016), Kenntnisse organisatorischer Art wie das Aufbrechen bestehender Datensilos und der ganzheitlichen, abteilungsübergreifenden Betrachtung von Datenketten sowie kulturelle Fähigkeiten, wie im Unternehmen eine Datenkultur aufgebaut und gelebt werden kann (Brownlow et al. 2015). Für die Entfaltung einer Wertrealisierung bedarf es neben dem Willen zur kontinuierlichen Anpassung und Verbesserung der Wertrealisierung auch neuer Methoden und Ansätze, um diese zu ermöglichen. Doch hier zeigt sich bei näherer Betrachtung eine inhaltliche Lücke im aktuellen Forschungsstand. Fruhwirth et al. (2020) arbeitet in einer umfassenden Literaturübersicht heraus, dass z.B. mit dem „Data Need Fit“ von Mathis und Köbler (2016), Managern ein Tool zur Analyse ihrer datengetriebenen Geschäftsmodelle an die Hand gegeben wurde sowie mit dem „data insight generator“ von Kühne und Böhmann (2019), ein Tool zum Aufzeigen der inhaltlichen Verbindung zwischen den Datenressourcen und den zu erzielenden Wertversprechen. Ferner existieren bereits etablierte, unterstützende Methoden zur Entwicklung von datengetriebenen Geschäftsmodellen, wie z. B. zur Auswahl von Erlösmodellen (Schüritz et al. 2017). Auch existieren mehrschrittige Vorgehensmodelle, z. B. zur Innovation und Implementierung von datengetriebenen Geschäftsmodellen (Hunke et al. 2017). Die Literaturanalyse zeigt jedoch auch die Notwendigkeit und den Bedarf nach einem Vorgehen zur nachhaltigen Umsetzung von datenbasierten Geschäftsmodellinnovationen auf. Die bestehenden Tools und methodischen Vorgehensmodelle beziehen sich vor allem auf Einzelaspekte, wie die Entscheidungsfindung oder das Ertragsmodell, unterstützen aber nur eingeschränkt die nachhaltige Umsetzung von datenbasierten Geschäftsmodellinnovationen und insbesondere die Realisierung von Werten aus diesen. Daher ist ein Vorgehensmodell, das insbesondere praktische Anforderungen bei der Implementierung in den Mittelpunkt rückt sowie wertrealisierende, aber auch wertmindernde Einflussfaktoren in sich verändernden Unternehmenskontexten berücksichtigt, von besonderem Interesse.

\section{Entwicklung eines Vorgehensmodells zur Wertrealisierung in datengetriebenen Geschäftsmodellen}

Die vorliegende Studie folgt dem Design Science Research, welches ein häufig angewendetes, problemlösungsorientiertes und etabliertes Forschungsparadigma darstellt (Hevner et al. 2004), das darauf abzielt neue Modelle oder Vorgehensmodelle zur praktischen Problemlösung zu entwickeln und dadurch neues Wissen zu generieren (Pries-Heje und Baskerville 2008). Dabei spielt die Entwicklung eine zentrale Be- 
deutung. Während ein Artefakt darauf abzielt, Lösungen für ein definiertes Problem anzubieten, dient die Evaluation der Qualitätsprüfung und Eignung des Artefakts für den Anwendungskontext. In Iterationszyklen entsteht durch Evaluation und Weiterentwicklung schließlich das finale Artefakt (Hevner et al. 2004). In dieser Studie wird als zentrales Artefakt prototypisch ein Vorgehensmodell entwickelt, das sich wiederum über Artefakte wie der Visualisierung von Problemen materialisiert und aus Interviews und Workshopmodulen zusammengesetzt ist. Designanforderungen spezifizieren dabei, welche Ergebnisse das Artefakt liefern muss und wann das Artefakt erfolgreich war. Im Folgenden werden der genauere Kontext sowie die Designanforderungen der durchgeführten Studie vorgestellt.

\subsection{Forschungsvorgehen zur Entwicklung des Vorgehensmodells}

Das in dieser Studie vorgestellte Vorgehensmodell wurde mit Hilfe von qualitativen Interviews sowie iterativ über prototypische Workshops entwickelt. Hierzu wurden zum einen Interviews mit allen späteren Workshop-Teilnehmern geführt, um die Einflussfaktoren für das zu entwickelnde Vorgehensmodell und die darin enthaltenen Workshopmodule zu eruieren. Darüber hinaus wurden Interviews mit fünf ausgewählten Methodenexperten durchgeführt, um ebenfalls Anforderungen aufzunehmen, aber auch um Hinweise für die Gestaltung des Artefakts zu erhalten. Das Vorgehensmodell wurde iterativ entwickelt und an die Anforderungen der interviewten Methodenexperten fortlaufend angepasst. Die durchgeführten Workshops dienten inhaltlich insbesondere zur Entwicklung eines Fahrplans zur Wertrealisierung in datengetriebenen Geschäftsmodellen. Dabei wurden die in den Interviews genannten Anforderungen anhand des prototypischen Vorgehensmodells evaluiert. In Summe setzt sich die Studie methodisch damit aus einer Anforderungsanalyse, der iterativen Entwicklung des Vorgehensmodells durch Methodenexperten und der Durchführung von Workshops zur Evaluation zusammen.

Die vorliegende Studie markiert den Schlusspunkt eines größer angelegten Forschungsprojekts. In diesem Projekt wurden beispielsweise in einem vorhergehenden Schritt 39 Interviews mit Experten zu datengetriebenen Geschäftsmodellen durchgeführt. Die Geschäftsmodelle der vorliegenden Studie waren in diesem Schritt ebenfalls bereits enthalten und wurden zum Teil über mehrere Jahre hinweg inhaltlich wie forschungsseitig begleitet. Die Erkenntnisse aus diesen Vorarbeiten flossen in den Aufbau der Studie ein, insbesondere in Form von praktischen Herausforderungen bzw. Anforderungen an ein umsetzbares und handhabbares Vorgehensmodell, welches sich auch über Abteilungs- und Fachgrenzen hinweg einsetzen lässt; welches auf die tatsächlich relevanten Einflussfaktoren für die Wertrealisierung von Daten fokussiert; und welches in einem dynamischen Kontext die pragmatische Priorisierung von Maßnahmen erlaubt.

In die Entwicklung des Vorgehensmodells sind Vorarbeiten vor allem in der Form eingeflossen, dass Workshops sowohl in der Forschung als auch in der Praxis etabliert sind, um auf Basis zuvor explorativ aufgedeckter Inhalte kollaborativ eine kontextuelle Problemlösung zu erarbeiten. Teilnehmer von Workshops haben dabei in der Regel bereits ein ausgeprägtes Verständnis für die zu erwartenden Workshopergebnisse. Diese Vorkenntnisse zeigen sich in einer aktiven Selbsteinbindung, 
Engagement und Kollaboration der Praxisteilnehmer und einer gemeinsamen Wissensbildung (Lain 2017; Ørngreen und Levinsen 2017), was im Rahmen des hier entwickelten Vorgehensmodells genutzt wurde. Alternative Vorgehen, beispielsweise die hierarchische Eskalation von Entscheidungspunkten waren aufgrund der fehlenden Linien- und/oder Projektverankerung (aufgrund der Neuartigkeit) nicht möglich.

\subsection{Forschungskontext der Geschäftsmodelle}

Analysegegenstand war die Begleitung von fünf datengetriebenen Geschäftsmodellen. Sie waren alle in einer frühen Entwicklungsphase ${ }^{1}$ und von strategischer Relevanz für das Unternehmen. Die Entwicklung fand unter Ressourcen- und Zeitlimitationen statt und erforderte die fachbereichsübergreifende Zusammenarbeit. Zur Beurteilung von Anforderungen war mitunter dediziertes Expertenwissen notwendig. In allen Geschäftsmodellen gab es Herausforderungen, die zum Scheitern des Geschäftsmodells hätten führen können. Inhaltlich handelte es sich bei den Geschäftsmodellen um einen Energieservice, zwei Mobilitätsservices, einen Kartenservice und einen Mobilitätsassistenten. Herausforderungen bestanden hinsichtlich der Vielschichtigkeit der Anforderungen aus den unterschiedlichsten Bereichen, der Priorisierung von Aktivitäten, der Allokation von Ressourcen, dem Umgang mit (zu) knappen Ressourcen und dem Bilden eines gemeinsamen Verständnisses. Diese Herausforderungen erscheinen auf den ersten Blick als wenig spezifisch für ein datengetriebenes Geschäftsmodell, sondern als eher typisch für ein sehr herausforderndes Projektumfeld. Auf den zweiten Blick fällt allerdings auf, dass beispielsweise Fähigkeiten der Data Analytics im derzeitigen Marktumfeld fast immer (zu) knapp sind, weil man die notwendige Fachkompetenz entweder gar nicht oder nur zu teuren Konditionen einkaufen kann. Im Kern bedeutet dies, dass in produktionsorientierten Unternehmen, der Unternehmensentwicklung folgend, verhältnismäßig weniger Data Analytics Kompetenzen vorhanden sind. Auch beim Bilden des gemeinsamen Verständnisses liefern datengetriebene Geschäftsmodelle besondere Herausforderungen: Eine streng arbeitsteilige Organisation der Arbeit wird durch die notwendige gleichzeitige Berücksichtigung von beispielsweise Compliance- und Datenschutzanforderungen, Geschäftsmodellaspekten wie technischer Möglichkeiten unmöglich gemacht. Hier sind Unternehmen in der Transition besonders gefordert, weil sie zur Bewältigung komplexer Produktsysteme einerseits höchste Qualitätsanforderungen sicherstellen müssen, aber gleichzeitig schnelle Anpassungen im Kontext stark vernetzter datengetriebener Geschäftsmodelle realisieren müssen. In Summe lässt sich festhalten, dass die heterogene Zusammensetzung der Beteiligten hinsichtlich Fachexpertise aber auch Zielsetzung ein herausforderndes Umfeld für die Weiterentwicklung der datengetriebenen Geschäftsmodelle darstellte.

\footnotetext{
1 Frühe Entwicklungsphase ist dadurch charakterisiert, dass das Geschäftsmodell initial konzipiert ist, bereits Ressourcen in die Umsetzung investiert wurden und ggfs. pilothaft Umsätze generiert wurden. Eine Integration in eine stabile Linien- und/oder Projektorganisation hat dabei noch nicht stattgefunden.
} 


\subsection{Forschungsvorgehen zur Evaluation des Vorgehensmodells}

Aus dem oben genannten Kontext der Entwicklung von datenbasierten Geschäftsmodellen ergibt sich, dass wertrealisierende und wertmindernde Einflussfaktoren besonderer Aufmerksamkeit bedürfen.

Wie bereits bei der Einführung der Forschungsmethode beschrieben, spielt unter anderem die Bewertung bzw. Evaluation des Artefakts, in unserem Fall des Vorgehensmodells, eine zentrale Rolle (z. B. Hevner et al. (2004)). Maßgeblich für die Evaluation war dabei die Technik nach March und Smith (1995). Sie stellten 1995 einen naturalistischen ex-post Evaluationszyklus vor, um entwickelte Methoden und Vorgehensmodelle nach klar definierten qualitativen Evaluationskriterien zu bewerten, insbesondere im IT-Kontext und unter Verwendung von Design Science. Häufig genannte Vorteile liegen im strukturierten und holistischen Rahmenwerk zur Evaluation von Design Science-Artefakten. In Design Science Research wird in diesem Kontext häufig von Designanforderungen gesprochen, insbesondere wenn diese vorab definiert werden, um das Artefakt im Nachgang zu evaluieren.

Insgesamt werden vier Evaluationskriterien unterschieden: Effizienz, Durchführbarkeit, Benutzerfreundlichkeit und Generalisierbarkeit. Die Effizienz wurde anhand des vorliegenden Kontexts der Studie spezifiziert, beispielsweise in der Praktikabilität (Prat et al. 2015) oder der Maßnahmenorientierung (Baumöl 2008). Die Durchführbarkeit und Benutzerfreundlichkeit beziehen sich z. B. auf die Wissensgenerierung oder das Fachbereichsverständnis, den Nutzerzugang und deren Akzeptanz sowie den kausalen und konsistenten Betrachtungscharakter (Ørngreen und Levinsen 2017). Schließlich bezieht sich die Generalisierbarkeit auf die Übertragbarkeit des Vorgehensmodells (Ahmed und Asraf 2018). Zusammenfassend liefern March und Smith (1995) ein wissenschaftlich fundiertes und in der Praxis erprobtes Rahmenwerk zur Integration spezifischer und kontextabhängiger Evaluationskriterien.

Die Evaluation des Vorgehensmodells wurde zweistufig durchgeführt. Einerseits wurden prozessuale Kriterien anhand von Beobachtungen dokumentiert und evaluiert. Ein Beispiel dafür ist das Kriterium der Praktikabilität: Konnten alle Teilnehmer den Vorgaben des Workshopleiters ohne Rückfragen problemlos und fehlerfrei folgen, wurde von einer hohen Praktikabilität ausgegangen. Andererseits kam der indikativen Beurteilung der Workshopteilnehmer unmittelbar nach Durchführung des Workshops eine große Bedeutung zu. So wurden am Ende der Workshops Feedbackrunden durchgeführt, die die genannten Evaluationskriterien abdeckten.

\section{Vorstellung des Vorgehensmodells zur Wertrealisierung in datengetriebenen Geschäftsmodellen}

\subsection{Design des Vorgehensmodells}

Bei dem entwickelten Vorgehensmodell handelt es sich um ein interview- und workshopbasiertes, systematisches Vorgehen bestehend aus fünf aufeinander folgenden Schritten. Diese fünf Schritte sind in zwei Abschnitte unterteilbar: einen initialen, explorativen Einzelinterviewabschnitt sowie einen sich anschließenden vier-schrittigen 
Workshopabschnitt. Die Einzelinterviews dauern jeweils $30 \mathrm{~min}$; für das sukzessive Durchlaufen der vier Workshopschritte ist ein Zeitrahmen von $90 \mathrm{~min}$ festgesetzt. Sowohl für die Interviews als auch für den Workshop ist die Einbindung von vier bis fünf relevanten Praxisteilnehmern, die bei der Entwicklung und Umsetzung des datengetriebenen Geschäftsmodells maßgeblich beteiligt waren, entscheidend.

Nachfolgend werden die fünf Schritte des Vorgehensmodells im Detail beispielhaft anhand eines der fünf datengetriebenen Geschäftsmodelle vorgestellt und erörtert. Es handelt sich hierbei um ein ,,analytics-as-a-service“-Geschäftsmodell im Bereich E-Mobilität (siehe Typisierung nach Hartmann et al. (2016)), bei dem analysierte Fahrzeug- und Energiedatenprodukte B2B-Kunden als Web-Service bereitgestellt werden. Für die Anwendung des entwickelten Vorgehensmodells nahmen vier Praxisteilnehmer aus den Bereichen IT-Strategie, Geschäftsfeldentwicklung und Data Science an den Einzelinterviews und dem Workshop teil.

\section{Schritt 1: Aufdeckung relevanter Einflussfaktoren auf die Wertrealisierung}

Die entscheidenden Einflussfaktoren auf die Wertrealisierung des datengetriebenen Geschäftsmodells werden in einem ersten Schritt in Einzelinterviews nach Flanagan (1954) exploriert. Die Praxisteilnehmer werden dabei gebeten, auf Basis ihrer aktuellen Erfahrung in der Umsetzung und dem Betrieb des datengetriebenen Geschäftsmodells, Einflussfaktoren zu erörtern, die die Wertrealisierung gefördert/ gestärkt bzw. gemindert oder gar teilweise verhindert haben (siehe Interviewfragen in Anhang A). Die Praxisteilnehmer sind angehalten, möglichst unvoreingenommen und offen zu sprechen und die Bedeutung der Einflussfaktoren auf die Wertrealisierung möglichst präzise darzulegen. Im Fokus steht dabei weniger die Anzahl als vielmehr die Bedeutung weniger Einflussfaktoren auf die Wertrealisierung. Nach Durchführung der Einzelinterviews erfolgt deren Auswertung und Verdichtung. Redundanzen werden entfernt. Beispielsweise wurden für das Geschäftsmodell im Bereich Energieservice acht Einflussfaktoren zur Förderung/Stärkung oder Verminderung der Wertrealisierung genannt, wie beispielhaft Partnerschaftsschulterblicke hinsichtlich eines gemeinsamen Business Cases, Risiko unterschiedlicher Datenqualitäten und der Bedarf nach Data Analytics Kompetenzen.

Schritt 2: Definition der, Wertrealisierung“ und, Wertminderung“ Im nächsten Schritt beginnt das Workshop-Setting. Es wird zunächst definiert, wie in dem datengetriebenen Geschäftsmodell Wert realisiert wird und welche Bedingungen, insbesondere hinsichtlich der verwendeten Daten, essentiell für die Wertrealisierung sind. Da bestimmte Einflussfaktoren nicht nur wertrealisierend sind, wird als Gegenpol auch die Wertminderung im datengetriebenen Geschäftsmodell konkret definiert. Ziel des ersten Schrittes ist es, Konsens unter den Teilnehmern zu erzeugen. Mit den Definitionen wird eine visuelle Skala zwischen den zwei Betrachtungspolen aufgespannt: von (-4) Wertminderung zu (+4) Wertrealisierung (siehe Abb. 1, Markierung (2)). Dies ermöglicht es Praxisteilnehmern, Einflussfaktoren im nächsten Schritt dediziert einzuordnen. Im Hinblick auf den Energieservice wurden als wertschaffende Aktivitäten, die Lieferung und Aufbereitung von Dateninformationen genannt, die erforderlich sind, um das Wertversprechen einer verlässlichen und umfassenden Dateninformationsbereitstellung als ,,analytics-as-a-service“ an B2B- 


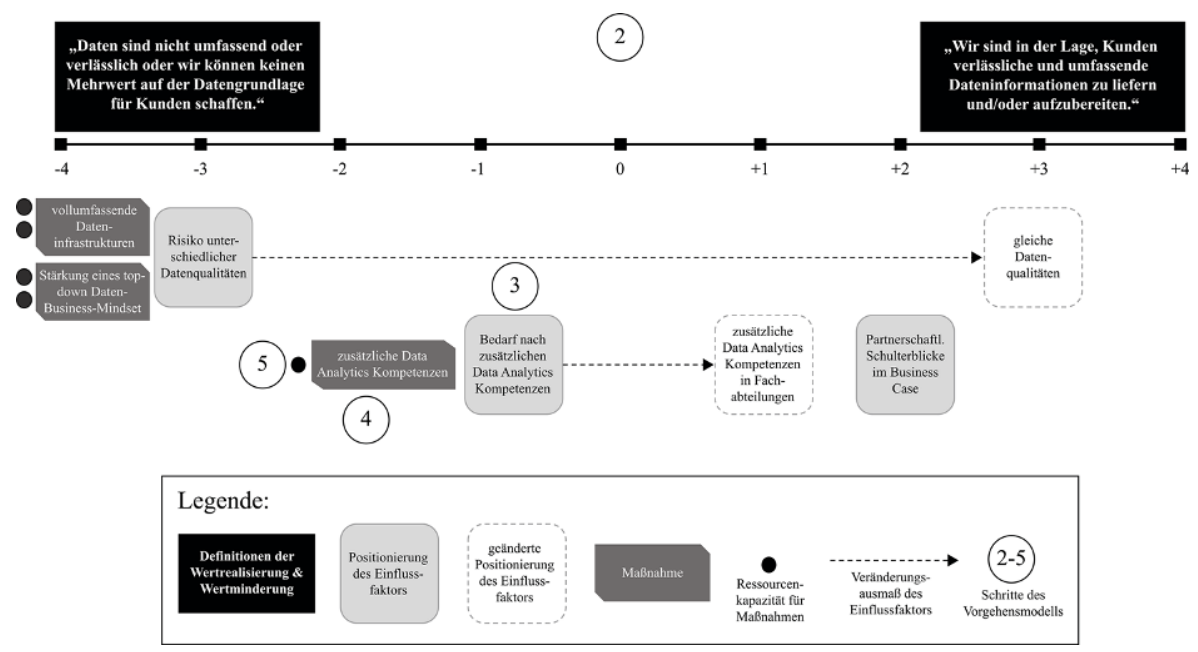

Abb. 1 Grafische Darstellung des Workshopabschnitts des Vorgehensmodells zur Wertrealisierung in datengetriebenen Geschäftsmodellen

Kunden zu erfüllen. Als Gegenpol „Wertminderung“ wurde der Fall genannt, wenn durch die Datenanalyseaktivitäten kein Mehrwert für Kunden geschaffen werden kann, z.B. durch eine nichtverlässliche oder nichtumfassende Dateninformationsbereitstellung.

Schritt 3: Einordnung der Einflussfaktoren auf die Wertrealisierung Nach Konsensbildung über welche Weise das datengetriebene Geschäftsmodell Wert realisiert und unter welchen Bedingungen Wert gemindert wird, werden die unter Schritt 1 hervorgebrachten, entscheidenden Einflussfaktoren auf die Wertrealisierung des datengetriebenen Geschäftsmodells verortet. Im Rahmen dieses Schrittes werden die Einflussfaktoren auf der Skala von -4 (Wertminderung) bis +4 (Wertrealisierung) eingeordnet (siehe Abb. 1, Markierung (3)). Ziel dieses Schrittes ist es, Wissen über die Stärke des Einflusses der einzelnen Faktoren auf die Wertrealisierung zu generieren und eine gemeinschaftliche Einordnung hierüber vorzunehmen. Für den Energieservice hat sich hierdurch herauskristallisiert, dass ein positiver Einflussfaktor auf die Wertrealisierung Partnerschaftsschulterblicke hinsichtlich eines gemeinsamen Business Cases (+2) ist. Als wertmindernd wurde hingegen das Risiko unterschiedlicher Datenqualitäten in den verteilten Datensystemen genannt (-3). Der Bedarf nach Data Analytics Kompetenzen wurde mit (-1) bewertet.

Schritt 4: Maßnahmenidentifizierung In Schritt 3 wurden Kenntnisse über die Einflussfaktoren und ihrer Wirkung auf Wertrealisierung und Wertminderung geschaffen. In Schritt 4 werden nun Maßnahmen identifiziert mit Hilfe derer entweder der Einfluss der wertmindernden Einflussfaktoren bestmöglich reduziert oder der Einfluss der wertrealisierenden Einflussfaktoren gefördert werden kann. Mehrere Maßnahmen für einen Einflussfaktor sind möglich. Als Prämisse der Maßnahmenentwicklung gilt, dass nur Maßnahmen entwickelt werden, die für das Unternehmen 
umsetzbar sind (z. B. hinsichtlich bestehender Kompetenzen oder Ressourcen). Ziel dieses Schrittes ist es, konkrete Maßnahmen zum Managen der Wertrealisierung zu identifizieren (siehe Abb. 1, Markierung (4)). Beim Energieservice sind beispielsweise für den wertmindernden Einflussfaktor Risiko unterschiedlicher Datenqualitäten (-3) zwei Maßnahmen identifiziert worden. Diese sind vollumfassende Dateninfrastrukturen und die Stärkung des vorhandenen top-down Daten-Business-Mindsets. Die erste Maßnahme erhöht die technische Leistbarkeit und reduziert damit das technische Risiko unterschiedlicher Datenqualitäten. Die Stärkung des vorhandenen top-down Daten-Business-Mindsets sichert eine nachhaltige Ressourcenallokation und Managementverpflichtung zur fortwährenden Optimierung der Datenqualität.

Schritt 5: Maßnahmenpriorisierung zur Umsetzung Im letzten Schritt werden die Maßnahmen unter der realistischen Bedingung limitierter Ressourcen reflektiert, bewertet und priorisiert. Hierzu werden symbolisch sieben „Ressourcenkapazitäten“ zur Verfügung gestellt. Diese gilt es entsprechend der Kapazitätsbedarfe der Maßnahmenumsetzung und des daraus resultierenden, möglichen Verbesserungsausmaßes der Wertrealisierung zu verteilen. Übergeordnetes Ziel dieses Schrittes ist es einen Konsens über die Maßnahmenpriorisierung zum Managen der Wertrealisierungen zu erarbeiten (siehe Abb. 1, Markierung (5)). Hierdurch wird ein holistisches Verständnis über die möglichen Maßnahmen und ihre Ressourcenkapazitätsbedarfe erzeugt. Im beispielhaften Anwendungsfall wurden vier der sieben verfügbaren Ressourcenkapazitäten in gleichen Teilen auf die beiden Maßnahmen vollumfassende Dateninfrastrukturen und die Stärkung des vorhandenen top-down Daten-BusinessMindsets verteilt. Die Allokation der vier Ressourcenkapazitäten verspricht eine große Hebelwirkung, um den wertmindernden Einflussfaktor $(-3)$ komplett aufzuheben und zukünftig zusätzlichen Wert $(+3)$ zu realisieren. Nach Einschätzung der Praxisteilnehmer heben beide Maßnahmen die wertmindernden Einflussfaktoren auf und schaffen Voraussetzungen, um aus dem datengetriebenen Geschäftsmodell verstärkt Wert zu realisieren. Des Weiteren wurde eine Ressourcenkapazität der Adressierung des identifizierten Bedarfs nach zusätzlichen Data Analytics Kompetenzen zugeordnet. Die zwei verbleibenden Ressourcenkapazitäten wurden nicht verteilt, da sie nur zu einer marginalen Verbesserung geführt hätten.

Abb. 1 fasst die vier Schritte des Workshopabschnitts grafisch zusammen; die Schritte sind mit (2-5) gekennzeichnet.

Wie die Ausführungen am Beispiel des Energieservices zeigen, unterstützt das entwickelte Vorgehensmodell dabei, die entscheidenden Einflussfaktoren auf die Wertrealisierung mit priorisierten Maßnahmen $\mathrm{zu}$ adressieren und ermöglicht es hierdurch, die Wertrealisierung des datengetriebenen Geschäftsmodells auf lange Sicht erfolgreich zu managen.

\subsection{Evaluation des Vorgehensmodells}

Im Folgenden wird das entwickelte Vorgehensmodell anhand der in Abschn. 3 vorgestellten Kriterien evaluiert. Die Beobachtungen sowie die Feedbackrunden haben ergeben, dass das Vorgehensmodell eine hohe Effizienz aufweist. Die systematische, zielgerichtete Vorgehensweise mit Fokussierung auf die Hebel der Wertrealisierung 
Tab. 1 Evaluation des Vorgehensmodells nach den Evaluationskriterien von March und Smith (1995)

\begin{tabular}{|c|c|c|}
\hline $\begin{array}{l}\text { Evaluations- } \\
\text { kriterien }\end{array}$ & Qualitative Rückmeldung zum Vorgehensmodell & $\begin{array}{l}\text { Grafische } \\
\text { Evaluati- } \\
\text { on }\end{array}$ \\
\hline Effizienz & $\begin{array}{l}\text { Alle Teilnehmer fanden einen direkten Einstieg } \\
\text { Konkrete Maßnahmen und ihre Hebel auf die Wertrealisierung wurden ge- } \\
\text { nannt } \\
\text { Eine weitere Unterteilung der Maßnahmen in konkrete Arbeitsschritte würde } \\
\text { die Effizienz zusätzlich steigern }\end{array}$ & \\
\hline $\begin{array}{l}\text { Durchführ- } \\
\text { barkeit }\end{array}$ & $\begin{array}{l}\text { Wissensgenerierung über Wertrealisierung und -minderung, Kausalzusam- } \\
\text { menhänge zwischen Einflussfaktoren und der Wertrealisierung wurden nach- } \\
\text { weislich erzielt } \\
\text { Gemeinsame Wahrnehmung und Sprache über entscheidende Einflussfakto- } \\
\text { ren, ihre Hebel und Maßnahmen zur Wertrealisierung wurden erzeugt } \\
\text { Gemeinsames Abschlussdokument mit Maßnahmendefinition und -priorisie- } \\
\text { rung wurde erzeugt } \\
\text { Systematisches Vorgehen (fünf Schritte) }\end{array}$ & \\
\hline $\begin{array}{l}\text { Benutzer- } \\
\text { freundlich- } \\
\text { keit }\end{array}$ & $\begin{array}{l}\text { Alle Teilnehmer waren über die } 90 \text { min selbstständig aktiv involviert } \\
\text { Es gab kaum Rückfragen bzgl. der Logik des Vorgehensmodells } \\
\text { Geringer Teilnehmeraufwand } \\
\text { ( } 30 \text { min pro Interview und } 90 \text { min für den Workshop) } \\
\text { Teilweise komplex, die Veränderungsausmaße der Wertrealisierung auf der } \\
\text { Skala von -4/+4 auszudrücken - jedoch über qualitative Verhältnisdarstel- } \\
\text { lung grundsätzlich allen möglich }\end{array}$ & \\
\hline $\begin{array}{l}\text { Generalisier- } \\
\text { barkeit }\end{array}$ & $\begin{array}{l}\text { Grundsätzlich möglich; aufgrund der spezifischen Fragestellungen im Work- } \\
\text { shop aber schwer bewertbar }\end{array}$ & \\
\hline
\end{tabular}

hat hohe Wirksamkeit, schnell gemeinschaftlich getragene Maßnahmen zur Verbesserung der Wertrealisierung zu definieren und zu priorisieren. Der Zeitaufwand zur Wissens- und Ergebnisgenerierung ist mit insgesamt 90 min für den Workshop und den initialen $4 \times 30$ min für die Einzelinterviews durchschnittlich effizient.

Das Vorgehensmodell weist eine gute Durchführbarkeit auf. Die Anforderung, Wertrealisierung handhabbar zu machen, wurde über die Einzelinterview- und Workshopabschnitte hinweg unter Einhaltung der gestellten Rahmenbedingungen erfüllt. Es wurden keine nicht gewollten Ergebnisse erzeugt und es kam zu keinen operativen Konflikten.

Die Anwendung des Vorgehensmodells hat über die Einzelinterviews und den Workshop hinweg zudem eine hohe Benutzerfreundlichkeit gezeigt. Alle Teilnehmer haben das Modell intuitiv richtig angewendet und waren fast durchgehend aktiv involviert. Der neuartige Zugang zu dem Thema hat zu positivem Feedback hinsichtlich der Nutzungserlebnisse geführt.

Trotz hohem Abstraktionsniveau und allgemeiner Übertragbarkeit der Logik des Vorgehensmodells auf andere Anwendungsfälle, ist die Generalisierbarkeit durchschnittlich zu bewerten. Im aktuellen Entwicklungsstand des Modells sind ein Großteil der praktischen Designanforderungen noch sehr unternehmens- und industriespezifisch.

Tab. 1 zeigt eine Auswahl qualitativer Rückmeldungen sowie eine grafische Veranschaulichung der Bewertung durch die Teilnehmer (in Form von „harvey balls“). 


\section{Diskussion und Implikationen}

Die vorliegende Studie hatte zum Ziel, Praktikern ein Vorgehensmodell an die Hand zu geben, um den Herausforderungen der Wertrealisierung datengetriebener Geschäftsmodelle begegnen und diese letztendlich erfolgreich implementieren zu können. Das Vorgehensmodell bietet einen ,greifbaren“ Ansatz, um die wertmindernden Einflussfaktoren (z.B. auf die Umsatzgenerierung) zu identifizieren und geeignete Maßnahmen zur Eliminierung oder Abschwächung dieser Einflussfaktoren zu entwickeln bzw. das Potenzial wertrealisierender Maßnahmen zu nutzen. Es zeigt u. a. auf, dass manche Maßnahmen einen hohen Ressourcenkapazitätsbedarf, aber nur einen geringen Hebel auf die Wertrealisierung, aufweisen; wohingegen andere Maßnahmen mit geringem Ressourcenkapazitätsbedarf einen großen Hebel besitzen. Zudem werden z. B. eine fachbereichsübergreifende Definition der Wertrealisierung, ein holistisches Verständnis über die entscheidenden Einflussfaktoren und ihre Hebelwirkungen auf die Wertrealisierung oder auch Kenntnisse über die kausalen Zusammenhänge zwischen den zu ergreifenden Maßnahmen und der Wertrealisierung entwickelt.

Die Studie adressiert damit den bisher wenig erforschten Untersuchungsgegenstand der nachhaltigen Umsetzung datengetriebener Geschäftsmodellinnovationen bei erfolgreicher Gestaltung der Wertrealisierung (siehe Fruhwirth et al. (2020)). Auch trägt die Studie zum aktuellen Forschungsstand der Methoden- und Vorgehensmodellfindung zum Aufzeigen und Erklären der inhaltlichen Zusammenhänge zwischen der Verwendung von Daten und der resultierenden Wertrealisierung datengetriebener Geschäftsmodelle bei (siehe z. B. Kühne und Böhmann (2019), Mathis und Köbler (2016) oder Zolnowski et al. (2016)).

Gleichzeitig weist das vorgestellte Vorgehensmodell auch einige Limitationen auf. Erstens, sind durch die fokussierte, empirische Untersuchung neben den Designanforderungen aus der Literatur insbesondere Praxisanforderungen eines Unternehmens berücksichtigt worden. Obwohl anzunehmen ist, dass vergleichbare Industrieunternehmen ähnliche Anforderungen stellen, ist die Generalisierbarkeit des Vorgehensmodells begrenzt (Gregor und Jones 2007). Zweitens, ist im Sinne der einfachen Anwendung die Ganzheitlichkeit und Reichhaltigkeit des Vorgehensmodells begrenzt (Prat et al. 2014). Bewusst wird auf die entscheidenden Hebel zur Wertrealisierung fokussiert. Dies beinhaltet jedoch das Risiko einer nicht vollständigen bzw. holistischen Betrachtung relevanter Aspekte zum Managen der Wertrealisierung. Drittens, wurde durch die zentrale Bewertungsskala zwar eine semiquantitative Bewertung vorgenommen; der Fokus liegt hierbei jedoch auf der qualitativen Einschätzung durch die Teilnehmer. Insofern wird die finanzielle Quantifizierbarkeit der Wertrealisierung im Rahmen des Vorgehensmodells nur bedingt unterstützt. Entsprechend bietet die Anwendung in einem breiteren, industrieübergreifenden Untersuchungsumfeld mit mehreren datengetriebenen Geschäftsmodellen sowie Ansätze zur finanziellen Quantifizierung der angepassten Wertrealisierung spannende Forschungsmöglichkeiten. Auch ein weiterer Evaluationszyklus, der die Maßnahmenumsetzung ex-post bewertet, hat methodisches Potenzial. 
Auf Basis der praktischen Implikationen kann das vorliegende Vorgehensmodell zusammenfassend als wichtiger Baustein für die Wertrealisierung und somit für die erfolgreiche Implementierung datengetriebener Geschäftsmodelle betrachtet werden.

\section{Anhang A}

\section{Interviewfragebogen aus Schritt 1}

1. Was waren entscheidende Einflussfaktoren, die die Wertrealisierung im datengetriebenen Geschäftsmodell gefördert/gestärkt bzw. gemindert oder gar teilweise verhindert haben?

2. Warum traten diese entscheidenden Einflussfaktoren auf?

3. Wie förderten/stärkten bzw. minderten oder gar verhinderten diese Einflussfaktoren die Wertrealisierung?

\section{Anhang B}

\section{Begleitmaterial der Workshops (Schritte 2-5)}

\section{Workshop Agenda}

Einführung in den Workshop

Vorgehensmodell Schritt 2:

Definition „Wertrealisierung“ \& „Wertminderung“ im datengetriebenen Geschäftsmodell

Vorgehensmodell Schritt 3:

Vorstellung und Detailbetrachtung der Einflussfaktoren aus den Interviews $\quad$ [10 Min]

Einordnung der Einflussfaktoren auf die Wertrealisierung $\quad$ [15 Min]

Vorgehensmodell Schritt 4:

Maßnahmenidentifizierung

Vorgehensmodell Schritt 5:

Maßnahmenpriorisierung zur Umsetzung

Abb. B.1 Workshop Agenda 


\section{Begleitfragen und -anweisungen zur Durchführung des Workshops}

\section{Vorgehensmodell Schritt 2:}

Wie zeigt sich Wertrealisierung und -minderung im datengetriebenen Geschäftsmodell (xyz)? Wie würdet ihr die Wertrealisierung und -minderung definieren? Überlegt und notiert euch bitte zuerst selbst Definitionen [ $5 \mathrm{Min}$ ], dann diskutiert eure Definitionen in der Gruppe und erstellt eine gemeinsame Definition [15 Min].

Vorgehensmodell Schritt 3:

Bitte ordnet gemeinsam die acht Einflussfaktoren horizontal auf der vorher definierten Achse (Wertrealisierung zu -minderung) entsprechend der Stärke ihres Einflusses auf die Achsenpole ein. Bitte diskutiert dabei auch mögliche Zusammenhänge der Einflussfaktoren zueinander und beachtet die Relationen der Einflussfaktoren bei der Einordung auf der Achse.

\section{Vorgehensmodell Schritt 4:}

Angenommen ihr bekommt den Auftrag die wertmindernden Einflussfaktoren zu verhindern/ einzuschränken und die wertrealisierenden Einflussfaktoren zu fördern, welche Maßnahmen müsstet ihr ergreifen? Überlegt und notiert euch bitte zuerst selbst geeignete Maßnahmen [ $5 \mathrm{Min}$ ], dann diskutiert die Maßnahmen in der Gruppe und klebt die finalen Maßnahmen, auf die ihr euch einigt habt, an die zu verändernden Einflussfaktoren auf dem Flipchart [15 Min].

\section{Vorgehensmodell Schritt 5:}

Angenommen ihr habt die sieben „Ressourcenkapazitäten“ zur Umsetzung der Maßnahmen und Verbesserung der Wertrealisierung zur Verfügung. Wie würdet ihr die Kapazitätspunkte verteilen? Berücksichtigt bitte hierbei die unterschiedliche Kapazitätsintensivität der Maßnahmen. Bitte diskutiert bei der Kapazitätsgewichtung auch den Zusammenhang zwischen Kapazitätsgewichtung und Ausmaß der Verbesserung der Wertrealisierung. Zeichnet anschließend die Kapazitätsgewichtung der Maßnahmen und das Ausmaß der möglichen Veränderung der Einflussfaktoren als Richtungspfeile auf der Achse ein. Stellt bitte anschließend euer finales Ergebnis vor.

Abb. B.2 Begleitfragen/-anweisungen zur Durchführung des Workshops

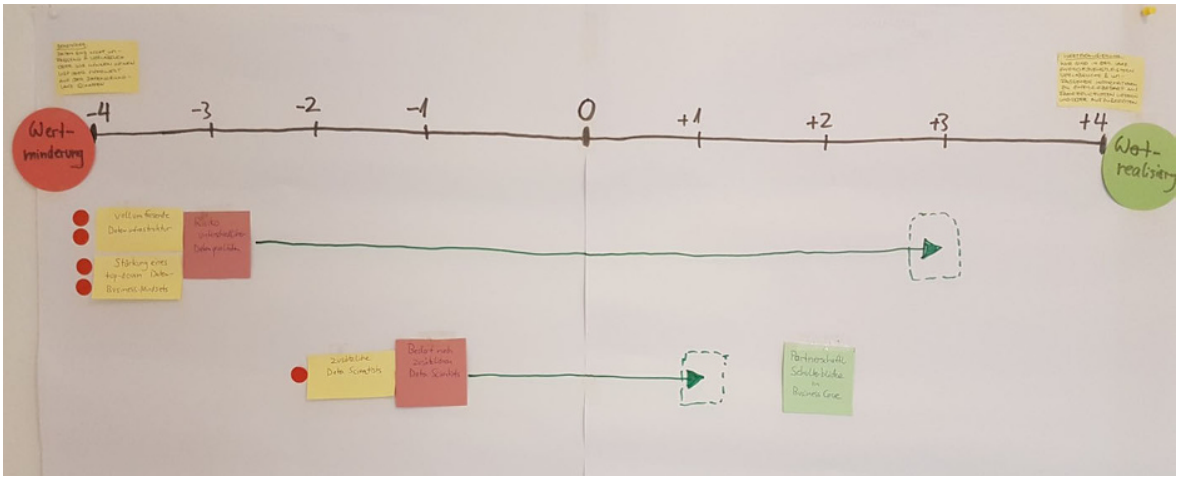

Abb. B.3 Ausschnitt aus Workshop - grafische Anwendung des Vorgehensmodells (Schritte 2-5)

Funding Open Access funding enabled and organized by Projekt DEAL.

Open Access Dieser Artikel wird unter der Creative Commons Namensnennung 4.0 International Lizenz veröffentlicht, welche die Nutzung, Vervielfältigung, Bearbeitung, Verbreitung und Wiedergabe in jeglichem Medium und Format erlaubt, sofern Sie den/die ursprünglichen Autor(en) und die Quelle ordnungsgemäß nennen, einen Link zur Creative Commons Lizenz beifügen und angeben, ob Änderungen vorgenommen wurden.

Die in diesem Artikel enthaltenen Bilder und sonstiges Drittmaterial unterliegen ebenfalls der genannten Creative Commons Lizenz, sofern sich aus der Abbildungslegende nichts anderes ergibt. Sofern das betreffende Material nicht unter der genannten Creative Commons Lizenz steht und die betreffende Handlung nicht nach gesetzlichen Vorschriften erlaubt ist, ist für die oben aufgeführten Weiterverwendungen des Materials die Einwilligung des jeweiligen Rechteinhabers einzuholen.

Weitere Details zur Lizenz entnehmen Sie bitte der Lizenzinformation auf http://creativecommons.org/ licenses/by/4.0/deed.de. 


\section{Literatur}

Ahmed S, Asraf RM (2018) The workshop as a qualitative research approach; lessons learnt from a "critical thinking through writing" workshop. Turkish Online J Des Art Commun. https://doi.org/10.7456/ 1080SSE/201

Akter S, Wamba SF (2016) Big data analytics in E-commerce: a systematic review and agenda for future research. Electron Markets 26:173-194. https://doi.org/10.1007/s12525-016-0219-0

Baumöl U (2008) Change Management in Organisationen. Gabler, Wiesbaden

Bosler M, Burr W, Ihring L (2018) Vernetzte Fahrzeuge; Empirische Analyse digitaler Geschäftsmodelle für Connected-Car-Services. HMD 55:329-348. https://doi.org/10.1365/s40702-018-0396-8

Brownlow J, Zaki M, Neely A, Urmetzer F (2015) Data and analytics - data-driven business models; a blueprint for innovation

Chen H, Chiang RHL, Storey VC (2012) Business intelligence and analytics; from big data to big impact. MISQ 36:1165-1188

Flanagan JC (1954) The critical incident technique. Psychol Bull 51:327-358

Fruhwirth M, Ropposch C, Pammer-Schindler V (2020) Supporting data-drive business model innovations; A structured literature review on tools and methods. J Bus Models 8:7-25. https://doi.org/10.5278/ ojs.jbm.v8i1.3529

Gregor S, Jones D (2007) The anatomy of a design theory. J Assoc Inf Syst 8:312-335

Günther W, Hosein M, Huysman M, Feldberg F (2017) Rushing or gold; tensions in creating and appropriating value from big data. Proceedings of the 38th International Conference on Information Systems. Bd. 38

Hartmann PM, Zaki M, Feldmann N, Neely A (2016) Capturing value from big data; a taxonomy of datadriven business models used by start-up firms. Int J Oper Prod Manag 36:1382-1406. https://doi.org/ 10.1108/IJOPM-02-2014-0098

Hevner AR, March SR, Park J, Ram S (2004) Design science in information systems research. MISQ 28:75-105

Hilbig R, Hecht S, Etsiwah B (2020) The rise of data-driven business models in the Berlin start-up scene. In: Barlatier P-J, Mention A-L (Hrsg) Managing digital open innovation. WORLD SCIENTIFIC, S 353-377

Hunke F, Schüritz R, Seebacher S, Illi A (2017) Towards a process model for data-driven business innovation. In: Proceedings of the 19th IEEE Conference on Business Informatics (CBI), S 150-157

Kühne B, Böhmann T (2019) Data-driven business models; building the bridge between data and value. In: Proceedings of the 27th European Conference on Infromation Systems (ECIS2019), Bd. 27

Kühne B, Zolnowski A, Bornholt J, Böhmann T (2019) Making data tangible for data-driven innovations in a business model context. Proceeding of the 25th American Conference on Information Systems. Bd. 25

Lain S (2017) Show, don't tell; Reading workshop fosters engagement and success. Tex J Lit Educ 5:160-167

March ST, Smith GF (1995) Design and natural science research on information technology. Decis Support Syst 15:251-266. https://doi.org/10.1016/0167-9236(94)00041-2

Mathis K, Köbler F (2016) Data-Need Fit-Towards data-driven business model innovation. Proceedings of the ServDes. 5th Service Design and Innovation conference. Bd. 125

Möller F, Stachon M, Hoffmann C, Bauhaus H, Otto B (2020) Data-driven business models in logistics; A taxonomy of optimization and visibility services. Proceedings of the 53rd Hawaii International Conference on System Sciences. Bd. 53, S 5379-5388

Oberländer AM, Übelhör J, Häckel B (2019) IIoT-basierte Geschäftsmodellinnovation im Industrie-Kontext: Archetypen und praktische Einblicke. HMD 56:1113-1125. https://doi.org/10.1365/s40702019-00570-1

Ørngreen R, Levinsen K (2017) Workshops as a research methodology. Electron J E Learn 15:70-81

Prat N, Comyn-Wattiau I, Cedric JA (2014) Artifact evaluation in information systems design-science research; A holistic view. Proceedings of the 23rd Pacific Asia Conference on Information Systems. Bd. 23

Prat N, Comyn-Wattiau I, Akoka J (2015) A Taxonomy of Evaluation Methods for Information Systems Artifacts. Journal of Management Information Systems 32(3):229-267

Pries-Heje B (2008) The design theory nexus. MISQ 32:731-755. https://doi.org/10.2307/25148870 
Schüritz R, Seebacher S, Dorner R (2017) Capturing value from data; revenue models for data-driven services. Proceedings of the 50th Hawaii International Conference on System Sciences. Bd. 50, S 5348-5357

Wiener M, Saunders C, Marabelli M (2020) Big-data business models; a critical literature review and multiperspective research framework. Int J Inf Technol 35:66-91. https://doi.org/10.1177/ 0268396219896811

Wixom BH, Ross JW (2017) How to monetize your data. MIT Sloan Manage Rev 58:10-13

Zolnowski A, Christiansen T, Jan G (2016) Business Model Transformation Patterns of Data-Driven Innovations. Proceedings of the 24th European Conference on Information Systems. 